\title{
Using mobile eye tracking to capture joint visual attention in collaborative experimentation
}

\author{
Sebastian Becker, Sergey Mukhametov, Philipp Pawels, and Jochen Kuhn \\ Department of Physics I Physics Education Research Group, \\ University of Kaiserslautern, Erwin-Schrödinger Str. 46, 67663 Kaiserslautern, Germany
}

\begin{abstract}
Experiments are of central importance for the natural sciences in general and in science education in particular, but the learning gains that teachers expect from them often fall short of expectations. This is especially true for student experiments, which are often conducted in dyads or small groups. In such a collaborative form of experimentation, the successful execution of the experiment and thus the achievement of the goals of the learning activity also depends on the cooperation of the students, so that a lack of learning success can also be caused by insufficient collaboration. In this study, mobile eye trackers were used with $N=40$ students to record gaze behavior during collaborative experimentation in the context of geometrical optics in order to investigate the influence of Joint Visual Attention (JVA) on learning success during experimentation. A significant relationship between JVA and learning gains was found for the setup phase with respect to experiment setup. The results show that especially during the setup of the experiment a successful collaboration of the experiment partners is of high importance for later successful execution of the experiment and thus support measures in this phase, such as the targeted directing of the attention of both experiment partners, could lead to an increase of the learning gain.
\end{abstract}

\footnotetext{
2021 PERC Proceedings edited by Bennett, Frank, and Vieyra; Peer-reviewed, doi.org/10.1119/perc.2021.pr.Becker Published by the American Association of Physics Teachers under a Creative Commons Attribution 4.0 license. Further distribution must maintain the cover page and attribution to the article's authors.
} 


\section{INTRODUCTION}

Laboratory learning experiments at school or university play a key role in science education [e.g., 1-3]. However, despite the acceptance of experiments as an integral part of the learning process, previous studies have shown that the potential for learning is only insufficiently exploited [4-9]. Since student experiments are often conducted in groups of two or three students, one possible reason for the lower learning success could be that the students do not collaborate sufficiently with each other. Since information is not available a priori in experiments, but must first be identified or generated in the experimental learning environment (e.g., by reading off measured values), coordination of visual attention should be essential for successful collaboration, which has already been empirically demonstrated for other learning activities, especially problem solving (e.g., [10-13]).

The processes associated with joint attention, i.e. with the simultaneous focusing of both participants' attention on the same aspect or object by means of different senses, is an important contribution to the study of collaborative activities. To investigate such joint attention processes in the selection and extraction of visual information during collaborative experimentation, wearable eye-tracking devices are suitable as a process-based research methodology. Special glasses (fig. 1) open up the possibility of capturing the experimenter's gaze data without restricting freedom of movement, so that experimental behavior remains unaffected by data collection.

In other contexts, mobile eye-tracking has already been successfully used to assess the quality of collaboration in joint problem-solving. For example, Schneider et al. used two mobile eye-trackers to capture levels of Joint Visual Attention of apprentices in logistics in co-located problem-solving settings and found that "joint attention can act as a proxy for students' quality of interaction" [10, p. 253].

Based on this approach, it is possible to investigate the interaction of the students to gain new insights into cooperative learning difficulties and their relation to learning success when conducting student experiments, which to our knowledge is still largely unexplored.

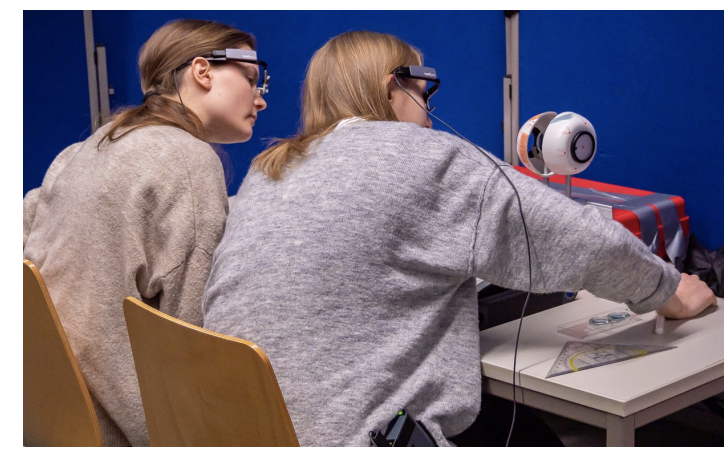

FIG. 1. Students with eye-tracking glasses (Tobii Pro Glasses 2).

\section{STATE OF RESEARCH}

Tomasello [14] states that Joint Attention (JA) is the tendency for social partners to focus on a common reference and furthermore, to monitor each other's attention to an external entity such as an object, a person or an event in various ways. The situation when two people concentrate on one element of the environment at the same time cannot be dubbed as joint attention. With the ability of JA, groups are able to form a common ground, take the perspective of their group members, build on their ideas, show some empathy or solve a problem in cooperation [15].

A subconstruct of JA is Joint Visual Attention (JVA), which occurs when two (or more) individuals look at the same spot at the same time. There is ample work showing that JVA is a central mechanism by which group members coordinate their actions and establish a common ground [e.g., 16, 17]. Therefore, JVA has been studied extensively by social and developmental psychologists and shown to be critical to many social interactions. As a finding of this research, there is now agreement in principle that JVA plays a central role in supporting students' conceptual convergence [18]. In this context, JVA can act as a proxy for the quality of students' collaboration, since without the ability to establish JVA, groups are unlikely to establish a common ground, take the perspective of their peers, build on their ideas, express some empathy or solve a problem together [10, p. 243]. In particular, it could be demonstrated that productive groups exhibit higher levels of JVA compared to less productive groups [11, 19, 20]. Against this background, Schneider et al. [10] even recognize JVA as one of the central constructs for any scientist interested in collaborative learning. According to Barron [21], JVA is seen as a prerequisite for virtually all social interactions. In his study, he was able to show that a group of students who did not achieve JVA more often ignored correct proposals and did not perform as well as similar groups.

Technological advancement enabled researchers to utilize (mobile) eye-tracker to capture JVA in collaborative situations. For example, Richardson and Dale [19] studied speaker-listener dyads and found that the higher the proportion of time that the speaker's and listener's eye movements were coupled, the better the listener performed on a subsequent comprehension test. Similarly, Jermann et al. [22] used two eye-trackers to capture gaze data to separate productive from less productive collaborative learning groups based on their common gaze patterns.

\section{RESEARCH QUESTIONS}

Based on the preliminary work presented, the goal of the study reported in this paper is to investigate the influence of JVA on learning outcomes in a collaborative experimental setting. Accordingly the main research questions of the study are as follows. 
RQ1: Is there a relationship between JVA and experimenters' learning success in collaborative experimentation?

Experimentation usually involves going through different phases, from setting up the experiment to evaluating the measurement results. Another research question of the study relates to the influence of JVA in these different experimentation phases.

RQ2: In what phases of experimentation does JVA have an impact on learning success?

\section{METHODOLOGY}

\section{A. Sample}

A total of 40 university students ( 21 female, 19 male) with a mean age of 22.3 years $(S D=3.2)$ participated in the study. All participants study health sciences at a technical university in Germany: 28 first-year, 1 second-year, 7 thirdyear, 1 fourth-year and 2 fifth-year (one missing data).

\section{B. Study}

The study was conducted as part of the two-week obligatory laboratory course on physical principles of physiological processes. Participants experimented in dyads, to which they were randomly assigned previously. The purpose of the experiment was to investigate the physiological features of the visual process using an optical bench (rail for rapid assembly of optical elements such as lenses, sensors and light sources), with optical components designed to imitate parts of the human eye. Throughout the experimental activity, the students wore mobile eye-trackers (Tobii Pro Glasses 2) with a sampling rate of $50 \mathrm{~Hz}$. Immediately before and after the experiment, a performance test was conducted to determine the learning gain from the experiment.

\section{Experiment setup}

Students interacted with an optical bench called the functional model of the human eye (fig.2). This model is designed to simulate the optical functions of the eye, such as the visualization of an object on the retina. It can also be a tool to simulate the accommodation of the eye (change in lens curvature), short-sightedness and long-sightedness. The model includes a half eye cup with an adjustable iris aperture, lens holder as well as 2 convex lenses ( $f=65 \mathrm{~mm}$ and $80 \mathrm{~mm}$ ), a half eye cup with a retina (transparent screen) as well as one correction lens each concave and convex, lens holder and experimental instruction.

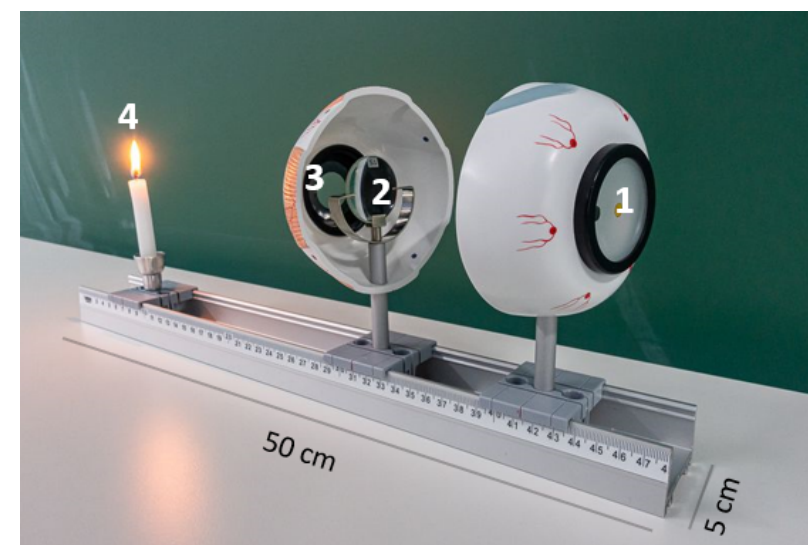

FIG. 2. The experimental setup: optical bench (slider with ruler markings and holders for optical parts) with the following optical elements: one half of the eye with a frosted glass pane (as a retina) (1), one half of the eye with a pupil attachment (2) and the adjustable iris aperture as the anterior segment of the eye (3), as well as a candle as the light source (4).

\section{Conducting the experiment}

Initially, the eye-tracking glasses were put on the test persons and then calibrated. All participants received the same instructions on how to handle the lenses beforehand. Before starting the experiment, the experimental materials were covered, which allowed both subjects to start experimenting simultaneously as soon as the study leader removed the cover. In order not to influence the collaboration between the experimenters in any way, the study leader then left the room for the duration of the experiment. The students received instructions on how to carry out and evaluate the experiment. The average duration of the experiment was 24.4 minutes $(S D=4.1)$.

The experimentation process comprised three phases:

- Phase 1: Set up the experiment and adjust the distance between the light source and the pupil so that an image is clearly visible on the simulated retina.

- Phase 2: Installation of a second lens and adjustment of the distance between the light source and the pupil.

- Phase 3: Finally, the participants had to simulate a short-sighted eye with the model and look for solutions to optimise the beam path by using diverging lens as glasses.

\section{Data collection and analysis}

\section{Learning gain}

Learning gain was measured by a pre-post performance test with six items in single-choice format by subtracting the score of the pre-test from the score of the post-test. The items were constructed based on known student conceptions from 
the literature [23] and the content validity was confirmed by a survey of experienced teachers and experts in the field of geometrical optics. Three of the items have already been used in a larger-scale study with $N=256$ students (Cronbach's alpha $=.80)$, the other three items were supplemented in accordance with the experiment conducted. One of the questions of the test, for example, was the following one: "A luminous object is imaged sharply onto a screen using a converging lens. Then the screen is moved away from the lens. What happens to the image on the screen?" The relevant response options were as follows: "A: The image on the screen becomes smaller", "B: The image on the screen remains the same size and becomes unfocused", "C: The image on the screen becomes larger and unfocused", "D: The image on the screen remains exactly the same, except that the screen is now further away".

\section{Gaze data}

a. Synchronization To determine the JVA from the gaze data of the experimental partners, the gaze data were manually synchronized in post-processing. Visual and acoustic triggers, such as revealing the experiment setup at the beginning of the experiment, were used to synchronize the gaze data on the one hand and to divide the entire experiment into individual experiment phases on the other by placing time markers in the recordings. The phases of the experiment were the same for both participants, as they worked together, and were determined by the need to first assemble the model and then to work sequentially with the three worksheets.

b. Mapping In a first step, the raw gaze data from each participant was passed through an I-VT (Identification by Velocity Threshold) gaze filter included in the Tobii Pro Lab software to detect the eye movement types "fixations" and "saccades". Fixations are those times when our eyes essentially stop scanning about the scene, holding the central foveal vision in place so that the visual system can take in detailed information about what is being looked at. Saccades are rapid, ballistic movements of the eyes that abruptly change the point of fixation. The threshold was set to $100^{\circ} \mathrm{s}$, as recommended by the manufacturer for gaze data acquisition with eye tracking glasses. In the next step, the gaze data were overlayed on the first-person video footage to match fixations with AOIs (Areas of Interest: objects observed by the participant across the timeline of the experiment). The areas of interest were chosen so that they would include all of the individual parts of the model the participants interacted with. This process is called mapping and can be done with Tobii Pro Lab using special built-in tools. The basis for this is a reference image of the object (snapshot), on which a "map" of the person's gaze was outlined. Thus, we mapped the recordings for all 40 participants with a total duration of more than 17 hours. Since the built-in automatic mapping can be inaccurate in some cases, all mapping results have been checked and corrected manually. Using the standard data export func- tion of the Tobii software, we obtained the initial data for the analysis of JVA.

c. Extraction of JVA From the synchronized and mapped gaze data of the experimental partners, in the next step, the time duration during which both subjects fixate the same AOI was determined for specific time intervals (separate phases and the duration of the entire experiment) using cross recurrence graphs (see fig. 3). The JVA value was calculated as the proportion of this time duration relative to the duration of the entire time interval, for which the JVA needs to be calculated. In a cross recurrence graph, the x-axis (y-axis) represents the time for the first (second) participant and pixels label moments of joint attention. Pixels along the main diagonal indicate that both experimental partners focused their attention on the same area at the same time, in our case either on the experimental setup (red pixels) or the task sheet (black pixels).

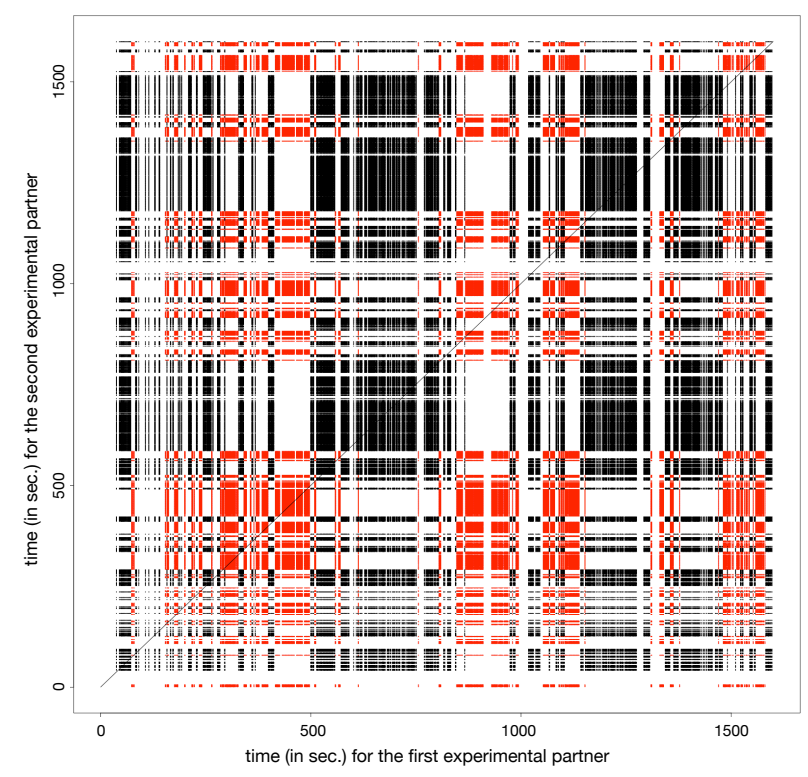

FIG. 3. Example cross recurrence graph of one dyad; coloured pixels along the diagonal indicate moments of joint attention on experimental setup (red) or task sheet (black).

\section{RESULTS AND DISCUSSION}

In order to investigate the influence of JVA on the learning gains of the dyads, the first step was to divide the dyads into two groups: Those for which both experimental partners showed an increase in learning from the pre-test to the posttest and those for which no or a negative increase in learning was found (see tab. I). To make the results as unambiguously interpretable as possible, dyads in which only one partner showed a positive learning gain were not included in the analysis, which reduced the sample size to $N=22$ (pos. gain: $N_{1}=14$, neg. or no gain: $N_{2}=8$ ). After a check of the 
application requirements:

- no outliers

- normal distribution of the continuous variable: Shapiro-Wilk test, R-function: shapiro.test, Rpackage: stats (vs. 4.0.3), $.086 \leq p \leq .580$

- equal variances: Levene's test, R-function: leveneTest, R-package: car (vs. 3.0-10), .176 $\leq p \leq .884$

the JVA values determined for the remaining dyads were subjected to a point-biserial correlation analysis concerning learning gain, the results of which are shown in tab. II.

TABLE I. Overview of the learning gains, which are indicated for both experiment partners separated by a dash.

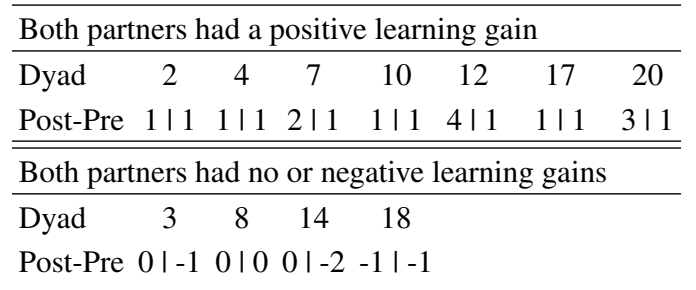

TABLE II. Results of the correlation analysis $\left(r_{p b i}\right.$ : point-biserial correlation coefficient; CI: $95 \%$ confidence interval; both calculated only if $\mathrm{p}<.050$.)

\begin{tabular}{lccc}
\hline Variable & $p$ & $r_{p b i}$ & CI \\
\hline Overall & .205 & - & - \\
Experiment setup & .006 & .763 & {$[.302 ; .935]$} \\
Task sheet & .050 & - & - \\
Experiment setup in phase 1 & .025 & .668 & {$[.113 ; .905]$} \\
Experiment setup in phase 2 & .105 & - & - \\
Experiment setup in phase 3 & .500 & - & - \\
\hline
\end{tabular}

Sample size: $N=22$ (pos. gain: $N_{1}=14$, neg. or no gain: $N_{2}=8$ )

When considering the JVA global, i.e., undifferentiated over the entire experimentation process, no correlation with the learning gain of the dyads can be found. However, when JVA is differentiated with respect to visual attention to the experiment setup and task sheet, there is a significant correlation between JVA and experimenter learning gains. This is an indication that attention on the same components of the experiment setup at the same time positively influences the experimental learning process. Differentiation of the JVA concerning the experimentation phases shows that especially the build-up phase at the beginning of the experimentation is of high importance for this, a significant correlation of the JVA with the learning gain can be proven for this phase.

\section{CONCLUSION AND OUTLOOK}

This first study aimed to capture JVA in a collaborative experimental setting using mobile eye trackers and to inter- rogate their influence on learning gains through the experiment. Thus, on the one hand, we succeeded in transferring the research methodology for investigating a learning activity essential in the natural sciences, experimentation. On the other hand, we have also succeeded in gaining initial insights into the influence of JVA on learning gain. Although no significant correlation could be demonstrated between the JVA for the overall experimentation process and learning gain, it could be demonstrated for the JVA concerning experiment setup, especially in the setup phase. This gives an indication of possible causes for a lack of learning success in collaborative experimentation and thus opens up possibilities for targeted support of the experimenters, for example, by directing the visual attention of the experimenting partners to the experiment setup in order to improve collaboration during experimentation.

The collection of process-based data such as JVA offers a great advantage over product-based data such as performance tests that it would be possible to support the experimentation process even before the experiment is completed. In many cases, these kinds of experiential learning sessions are conducted without the help and guidance of an instructor, and the success of the experimental work depends entirely on the success of the collaboration between the participants involved in the learning process. Therefore, identifying the quality of the collaborative process during its development, a comparative analysis of these kinds of experiments conducted with or without guidance, as well as understanding what instruction can improve the cooperation, are promising options for the development of this research. In perspective, the research methodology thus enables quantification of the quality of collaboration already during experimentation. The data obtained in this way could in turn be used either by the teacher $\mathrm{him} /$ herself or by a cognitive system for adaptive support of the experimentation process.

It should be noted, nevertheless, that this study is the first to use a new research methodology to investigate the collaborative experimentation process, but that the data analysis is based on a small sample. The results of this study thus provide only first indications of a positive influence of JVA on learning success in collaborative experimentation. However, this possible influence should first be statistically verified in follow-up studies with a larger sample size before a corresponding conclusion can be drawn. JVA can be used to look in detail at role-taking within mini-groups, as well as to estimate cognitive expenses on transactional costs. As these parameters are fundamental to the consideration of collaborative cognitive load [24-26], such studies may provide an opportunity to establish the relationship between JVA and students' cognitive load specifically in the case of collaborative learning activities involving experimentation. Another possible line of research is the additional collection of audio data to provide insights into the communication process during collaborative experimentation, which would allow triangulation of the JVA data with respect to the quality of the collaboration. 
[1] D. L. Haury and P. Rillero, Perspectives of Hands-On Science Teaching (ERIC Clearinghouse for Science, Mathematics and Environmental Education, Columbus, OH, 1994).

[2] C. Haagen-Schützenhöfer and B. Joham, Professionalising Physics Teachers in Doing Experimental Work, CEPS Journal 8, 9 (2018).

[3] B. E. Woolnough, The role of the laboratory in physics education, Physics Education 14, 70 (1979).

[4] A. Hofstein and V. N. Lunetta, The laboratory in science education: Foundations for the twenty-first century, Science Education, Science Education 88, 28 (2004).

[5] S. J. Husnaini and S. Chen, Effects of guided inquiry virtual and physical laboratories on conceptual understanding, inquiry performance, scientific inquiry self-efficacy, and enjoyment, Physical Review Physics Education Research 15, 010119 (2019).

[6] H. O. Kapici, H. Akcay, and T. de Jong, Using hands-on and virtual laboratories alone or together-which works better for acquiring knowledge and skills?, Journal of Science Education and Technology 28, 231 (2019).

[7] P. A. Kirschner and M. A. M. Meester, The laboratory in higher science education: Problems, premises and objectives, Higher Education 17, 81 (1988).

[8] T. S. Volkwyn, S. Allie, A. Buffler, and F. Lubben, Impact of a conventional introductory laboratory course on the understanding of measurement, Physical Review Special Topics - Physics Education Research 4, 010108 (2008).

[9] C. Wieman and N. G. Holmes, Measuring the impact of an instructional laboratory on the learning of introductory physics, American Journal of Physics, American Journal of Physics 83, 972 (2015).

[10] B. Schneider, K. Sharma, S. Cuendet, G. Zufferey, P. Dillenbourg, and R. Pea, Leveraging mobile eye-trackers to capture joint visual attention in co-located collaborative learning groups, International Journal of Computer-Supported Collaborative Learning 13, 241 (2018).

[11] B. Schneider and R. Pea, Real-time mutual gaze perception enhances collaborative learning and collaboration quality., International Journal of Computer-Supported Collaborative Learning 8, 375 (2013).

[12] K. Sharma, J. K. Olsen, V. Aleven, and N. Rummel, Measuring causality between collaborative and individual gaze metrics for collaborative problem-solving with intelligent tutoring systems, Journal of Computer Assisted Learning 37, 51 (2021).

[13] M. M. Villamor and M. M. T. Rodrigo, Gaze collaboration patterns of successful and unsuccessful programming pairs using cross-recurrence quantification analysis, Research and Practice in Technology Enhanced Learning 14, 10.1186/s41039-0190118-z (2019).
[14] M. Tomasello, Joint attention as social cognition, in Joint Attention: Its Origins and Role in Development, edited by P. J. Moore, C. Dunham (Lawrence Erlbaum Associates, Inc., Hillsdale, NJ, 1995) pp. 103-130.

[15] P. Mundy, M. Sigman, and C. Kasari, A longitudinal study of joint attention and language development in autistic children., Journal of Autism and Developmental Disorders 20, 115 (1990).

[16] H. H. Clark and S. E. Brennan, Grounding in communication., Perspectives on socially shared cognition., , 127 (1991).

[17] H. H. Clark, Language use and language users, in Handbook of social psychology, edited by E. Lindzey, G Aronson (Harper and Row, New York, NY, USA, 1985) pp. 179-231, 3rd ed.

[18] J. Roschelle, Learning by collaborating: Convergent conceptual change, Journal of the Learning Sciences, Journal of the Learning Sciences 2, 235 (1992).

[19] D. C. Richardson and R. Dale, Looking to understand: the coupling between speakers' and listeners' eye movements and its relationship to discourse comprehension., Cogn Sci 29, 1045 (2005).

[20] D. Gergle and A. T. Clark, See what i'm saying? using dyadic mobile eye tracking to study collaborative reference, in Proceedings of the ACM 2011 Conference on Computer Supported Cooperative Work, CSCW '11 (Association for Computing Machinery, New York, NY, USA, 2011) pp. 435-444.

[21] B. Barron, Achieving coordination in collaborative problemsolving groups, Journal of the Learning Sciences, Journal of the Learning Sciences 9, 403 (2000).

[22] P. Jermann, D. Mullins, M.-A. Nüssli, and P. Dillenbourg, Collaborative gaze footprints: Correlates of interaction quality, CSCL2011 Conference Proceedings. Volume I - Long Papers (2011).

[23] C. Haagen-Schützenhöfer and M. Hopf, Schülervorstellungen zur geometrischen Optik [Student conceptions of geometric optics], in Schülervorstellungen und Physikunterricht: Ein Lehrbuch für Studium, Referendariat und Unterrichtspraxis [Student conceptions and physics education: a textbook for study, teacher training, and teaching practice], edited by H. Schecker, T. Wilhelm, M. Hopf, and R. Duit (Springer Berlin Heidelberg, Berlin, Heidelberg, 2018) pp. 89-114.

[24] J. Janssen and P. A. Kirschner, Applying collaborative cognitive load theory to computer-supported collaborative learning: towards a research agenda, Educational Technology Research and Development 68, 783 (2020).

[25] I. T. Skuballa, K. M. Xu, and H. Jarodzka, The impact of coactors on cognitive load: When the mere presence of others makes learning more difficult, Computers in Human Behavior 101, 30 (2019).

[26] P. A. Kirschner, J. Sweller, F. Kirschner, and J. Zambrano R., From cognitive load theory to collaborative cognitive load theory, International Journal of Computer-Supported Collaborative Learning 13, 213 (2018). 